Published in final edited form as:

Behav Sleep Med. ; : 1-13. doi:10.1080/15402002.2018.1546709.

\title{
Effects of Cognitive Behavioral Therapy for Insomnia on Sleep, Symptoms, Stress and Autonomic Function among Patients with Heart Failure
}

\author{
Samantha Conley, PhD, \\ Yale School of Nursing \\ George Anderson, PhD, \\ Yale Child Study Center \\ John Cline, PhD, \\ Yale School of Medicine \\ Laura Andrews, PhD, RN, \\ Yale School of Nursing \\ Vahid Mohsenin, MD, \\ Yale School of Medicine \\ Daniel Jacoby, MD, and \\ Yale School of Medicine \\ Sangchoon Jeon, PhD \\ Yale School of Nursing
}

Nancy S. Redeker, PhD, RN, FAHA, FAAN [Beatrice Renfield Term Professor of Nursing], Yale School of Nursing, PO Box 27399, West Haven, CT 06516-7399, 203-785-6437

\section{Abstract}

Background: Insomnia is common among patients with stable heart failure (HF) and associated with inflammation and altered autonomic function.

Purpose: The purposes of this study were to examine the effects of cognitive behavioral therapy for insomnia (CBT-I) on the Hypothalamic Pituitary (HPA) Axis, autonomic function, inflammation and circadian rhythmicity and the associations between these biomarkers and insomnia, sleep characteristics, symptoms, functional performance, and sleep-related cognitions.

Methods: We conducted a sub-analysis of a pilot randomized controlled trial (RCT) (NCT02827799) whose primary aim was to test the effects of CBT-I on insomnia. We randomized 51 patients with stable Class II-IV HF to CBT-I $(n=30)$ or attention control $(n=21)$. Participants completed wrist actigraphy and self-reported insomnia severity, sleep characteristics, sleep-related cognitions, daytime symptoms, and functional performance. We measured day and nighttime urinary free cortisol, melatonin sulfate, epinephrine and norepinephrine at baseline and 2 weeks after CBT-I and computed general linear models and partial correlations.

Results: CBT-I had no effects on the biomarkers, but there were statistically significant negative cross-sectional correlations between the ratio of day/night urinary free cortisol and sleep 
disturbance, anxiety, fatigue, depression, and negative sleep cognitions. Increases in the ratio of day/night cortisol were associated with statistically significant improvements in fatigue, depression, sleep duration and sleep-related cognitions.

Conclusions: Biomarkers of stress and autonomic function are associated with sleep, sleeprelated symptoms and cognitions among people with chronic HF. Future studies are needed to identify potential causal relationships and the impact of sleep interventions.

\section{Keywords}

insomnia; stress; actigraphy; cortisol; melatonin

\section{Introduction}

As many as $25-56 \%$ of patients with chronic heart failure (HF), a group that includes over 6.5 million Americans (Benjamin et al., 2017) and many others worldwide, suffer from chronic insomnia (Gau, Chen, Wu, Lin, \& Chao; Redeker, Jeon, et al., 2010; Redeker \& Stein, 2006). Recent studies revealed that insomnia predicted incident HF and death over 11 years (Laugsand, Strand, Platou, Vatten, \& Janszky, 2014), and both insomnia and HF are associated with activation of the renin-angiotensin-aldosterone system (Kanno et al., 2016) and inflammation (Ahmad, Fiuzat, Felker, \& O'Connor, 2012). These findings emphasize the importance of understanding the biological mechanisms that may underlie the associations between insomnia and $\mathrm{HF}$ and the biological consequences of insomnia treatment in these patients because insomnia may worsen HF outcomes, and it is possible that treatment of insomnia may improve these outcomes.

Among healthy people, sleep onset is associated with declines in circulating catecholamines and decreased cortisol (Basta, Chrousos, Vela-Bueno, \& Vgontzas, 2007; Carroll et al., 2015). Insomnia, a disorder of psychophysiological arousal, is associated with elevated stress levels (Pereg et al., 2013), chronic activation of the hypothalamic-pituitary adrenal (HPA) axis (Meerlo, Sgoifo, \& Suchecki, 2008; Vgontzas \& Chrousos, 2002; Vgontzas et al., 2002), autonomic dysfunction, somatic and central nervous system hyperarousal (Basta et al., 2007; M. T. Smith, Huang, \& Manber, 2005). Circadian alterations in these biomarkers in some (Backhaus, Junghanns, \& Hohagen, 2004; Gallucci et al., 2014; Lack, Gradisar, Van Someren, Wright, \& Lushington, 2008), but not all (Basta et al., 2007) studies.

Insomnia, but not sleep disordered breathing, was associated with symptoms such as fatigue, depression, and excessive daytime sleepiness among patients with stable HF who received care in specialized HF disease management programs (Jeon \& Redeker, 2016; Redeker, Jeon, et al., 2010; Redeker, Muench, et al., 2010), and these daytime symptoms are associated with altered biological processes. For example, depression is associated with disrupted circadian rhythmicity, decreased melatonin, and increased cortisol. However, little is known about the associations among insomnia, daytime symptoms and biomarkers of circadian rhythmicity, inflammation and HPA axis function among people with HF who have high levels of symptom burden. 
Cognitive behavioral therapy for insomnia (CBT-I) targets dysfunctional cognitions and behaviors that perpetuate insomnia. Although a large body of science supports the effects of CBT-I on sleep characteristics, recent studies suggest that it may also improve the biology of insomnia. For example, CBT-I reduced C-reactive protein (CRP), inflammatory cytokines, and pro-inflammatory gene expression beginning 4 months post-CBT-I in older adults, among whom a large proportion had cardiovascular disease (Irwin et al., 2015; Irwin et al., 2014). Sleep restriction, a component of CBT-I, decreased early morning cortisol in patients on peritoneal dialysis (Miller et al., 2015) and improved IFN-gamma in breast cancer survivors (Savard, Simard, Ivers, \& Morin, 2005), but the direct biological effects of CBT-I or the effects of improvements in insomnia and sleep characteristics on inflammatory or other biomarkers have not been systematically examined in HF patients.

The aims of the current study were to examine the (1) effects of CBT-I on biomarkers reflecting the HPA axis (urinary free cortisol), circadian rhythms (urinary melatonin sulfate) and the autonomic nervous system (urinary catecholamines); (2) associations between insomnia, objective and self-reported sleep characteristics, sleep-related daytime symptoms and urinary free cortisol, melatonin sulfate, and catecholamines; and (3) associations between cognitive arousal (i.e., dysfunctional beliefs and attitudes about sleep) and urinary free cortisol, melatonin sulfate, and catecholamines among patients with stable HF who had insomnia at baseline.

\section{METHODS}

\section{Design}

We conducted a pilot randomized controlled trial (RCT) (NIH R21NR011387; NCT02827799). The primary aim of the parent study was to test the effects of CBT-I on insomnia and fatigue (Redeker et al., 2015), and we found statistically large effects on these outcomes and sleep-related cognitions (Redeker et al., 2017). We randomized HF patients in groups to four weeks of bi-weekly group-based CBT-I over eight weeks or an attention control condition (HF self-management education) provided in the same format. We collected measures of sleep, symptoms, and biomarkers at baseline and at 2 weeks postCBT-I. We obtained human subjects approval, and all participants provided written informed consent.

\section{Sample}

We recruited participants from a HF disease management program who were aged 18 years of age or older, lived at home, cognitively intact by clinical impression and had New York Heart Association Functional Classification II-IV HF, and an Insomnia Severity Index > 7, (Bastien, Vallieres, \& Morin, 2001), indicating at least mild chronic insomnia. We did not include ejection fraction as an enrollment criterion because it and the presence or absence of systolic dysfunction were not related to insomnia in our previous study (Redeker, Jeon, et al., 2010). Patients were excluded if they had untreated sleep disordered breathing (Apnea Hypopnea Index 210), based on Apnea Risk Evaluation Screening, conducted as part of this study (ARES, Watermark Medical, Inc.) or a previous diagnosis of sleep disordered breathing, but patients who had sleep disordered breathing and were adherent to positive 
airway pressure therapy were included. Full details of the screening for sleep apnea were previously reported (Redeker et al., 2015). We excluded patients with narcolepsy, restless legs syndrome, end-stage renal failure, seizure disorders, severe excessive daytime sleepiness (Epworth Sleepiness Scores > 18), or had neurological or musculoskeletal disorders that restricted the motion of the non-dominant arm (due to the possible confounding effects on wrist actigraph data).

Statistical power was not a primary rationale for the sample size of the original study designed to evaluate preliminary efficacy, acceptability, and feasibility (Redeker et al., 2015). Due to the exploratory nature of this analysis we did not calculate a priori power for the biomarker analyses.

\section{Procedures}

We randomized participants in groups to either CBT-I or the attention-control condition. The rationale for the content, format, and duration of the treatment conditions have previously been reported in detail (Redeker et al., 2015). In brief, the CBT-I sessions included stimulus control, sleep hygiene instruction, sleep restriction, cognitive therapy, progressive muscle relaxation, and optional hypnotic tapering provided by a clinical psychologist sleep specialist. Four one-hour sessions were scheduled bi-weekly, with a telephone call on the alternate weeks (total eight weeks) (Redeker et al., 2015). This duration and frequency were based on a previous study of dose effects of CBT-I (Edinger, Wohlgemuth, Radtke, Coffman, \& Carney, 2007). The attention control condition consisted of HF self-management education, including guidance on symptom monitoring, medication adherence, diet and fluid monitoring, physical activity, and sleep hygiene education and was designed to have consistent format, time and attention to the CBT-I condition. We provided the written materials from the HF self-management class to the patients in the CBT-I condition. An advanced practice registered nurse provided the attention control condition and followed the same timing of sessions as the CBT-I intervention.

\section{Variables and Measures}

Participants completed self-report measures of insomnia, sleep characteristics, sleep-related symptoms, dysfunctional beliefs and attitudes about sleep, and 2 weeks of wrist actigraphy and sleep diaries. As detailed below, daytime and nocturnal urinary specimens were obtained. Participants completed all measures at baseline and 2 weeks after the conclusion of the intervention.

Clinical and demographic variables.-We elicited demographic and clinical characteristics of the sample, including medical history and the Charlson Comorbidity Index (Charlson, Pompei, Ales, \& MacKenzie, 1987) and the New York Heart Association Functional Classification.

Biological variables included urinary norepinephrine, epinephrine, cortisol, melatonin sulfate, and creatinine. Urine samples were obtained between 1PM - 5 PM in the afternoon and again in the morning following the overnight sleep period to permit analysis of diurnal (day-night) variation. Participants were instructed to empty their bladders at bedtime, 
discard the urine and then void into the container through the night and in the morning (to reflect nocturnal biomarkers levels over the entire night). They then emptied their bladders prior to the beginning of the 1-5 PM data collection and then saved the urine obtained between 1-5 PM. The total urine volumes were measured, and five $2.0 \mathrm{ml}$ aliquots were stored at $-80 \mathrm{C}$ until analyzed.

Urinary free norepinephrine and epinephrine were determined after alumina extraction and separation by reverse phase ion-pair high performance liquid chromatography according to published methods. (Anderson, Durkin, Morton, \& Cohen, 1988) and reported as nomograms/milligram ( $\mathrm{ng} / \mathrm{mg}$ ) of creatinine. The compounds were detected fluorometrically and determined with interassay coefficients of variation of 5-7\%.

Urinary free cortisol was determined by radioimmunoassay after methylene chloride extraction. The commercially available DPC (Los Angeles, CA) Coat-a-Count RIA kit was used with an inter-assay coefficient of variation of less than 10\%; measured cortisol levels were expressed as nanograms/milligram of creatinine.

Urinary melatonin sulfate was determined by radioimmunoassay (RIA) after dilution. A commercially available gas chromatography-mass spectrometry validated RIA kit supplied by Stockgrand Ltd. (Surrey, UK) with an interassay coefficient of variation of less than $10 \%$ was used. Levels of urinary creatinine were determined after dilution and direct injection on a reverse-phase high performance lipid chromatography system, with detection by ultraviolet absorbance (Tsikas, Wolf, \& Frolich, 2004). Quality assessment samples were run with each assay and quality control procedures were implemented as deemed appropriate by application of Westgard rules (Westgard, Barry, Hunt, \& Groth, 1981).

Sleep Characteristics.-To elicit objective attributes of sleep (quality, duration, continuity, latency) and patient perceptions about sleep (sleep quality, duration, continuity, insomnia severity), we obtained self-report and actigraph sleep measures. The Pittsburgh Sleep Quality Index (PSQI) (D. J. Buysse, Reynolds, Monk, Berman, \& Kupfer, 1989) elicits sleep efficiency, duration, latency, and global sleep quality and is reliable and valid (D. J. Buysse et al., 1989; D. J. Buysse et al., 1991). Global sleep quality ranges from 0 - 21 (higher score indicates poorer sleep quality; score $>5$ characterizes poor sleep quality). The PSQI has a sensitivity of $89.6 \%$ and specificity of $86.5 \%$ for "good" vs. "poor" sleep (D. J. Buysse et al., 1989). Cronbach's Alpha was 0.83 in HF patients (Redeker, Jeon, et al., 2010).

We used the Insomnia Severity Index (ISI) to evaluate insomnia severity. The ISI is internally consistent (0.74 - 0.88) (Bastien et al., 2001; Blais, Gendron, Mimeault, \& Morin, 1997) and sensitive to treatment (Bastien et al., 2001; Blais et al., 1997; Morin, Belleville, Belanger, \& Ivers, 2011; S. Smith \& Trinder, 2001) and reflects the ICSD-3 criteria for insomnia (Wong et al., 2017). Possible scores range from 0-28. Cronbach's alpha was .70 in our study.

Because of our interest in sleep occurring during everyday life in naturalistic environments, we measured objective sleep characteristics with the Respironics Minimitter Actiwatch AW-64, a wrist-worn accelerometer. Correlations between actigraph and polysomnographic 
(PSG) sleep variables are between 0.82-0.98 (sleep efficiency) and 0.90-0.97 (sleep duration) in normal sleepers (Benson et al., 2004; Blood, Sack, Percy, \& Pen, 1997; Edinger, 2004; Kushida et al., 2001). Actigraphy corresponded with PSG on sleep efficiency, awakenings, wake after sleep onset, and total sleep time among people with insomnia (Lichstein et al., 2006) and is sensitive to changes over time and treatment (Littner et al., 2003). However actigraphy is less concordant with PSG in people with very fragmented sleep due to its lower sensitivity to wakefulness than sleep (Sadeh, 2011). A daily sleep diary was used to assist in interpreting the wrist actigraph data (e.g., lights out time, lights on time). Diaries are reliable, valid and frequently used for this purpose.

Daytime Symptoms.-We measured sleep-related daytime symptoms experienced by HF patients (i.e., fatigue, excessive daytime sleepiness, anxiety, depression, and pain). The Multi-dimensional Assessment of Fatigue Index elicits severity, distress, degree of interference and timing of fatigue (Belza, 1990) has concurrent $(r=0.84)$ and divergent $(r=$ -0.62 ) validity, and internal consistency (alpha $=0.84)$ in HF patients (Redeker \& Hilkert, 2005).

The Epworth Sleepiness Scale (Johns, 1991) was used to measure self-reported daytime sleepiness. It is reliable in HF patients (coefficient alpha $=0.87)($ Redeker $\&$ Stein, 2006). The Center for the Epidemiological Studies Depression Scale (Devins \& Orme, 1985; Radloff \& Teri, 1986) was used to measure depressive symptoms. Cronbach's alpha was 0.83 in HF patients (Redeker \& Stein, 2006). The 40-item Spielberger State Inventory Form $\mathrm{Y}$ was used to measure state anxiety. Test-test reliability exceeds 0.7 (Spielberger, 1983).

We also used the Medical Outcomes Study SF-36v2 Physical Function Scale and the SF36v2 Bodily Pain scale. These instruments have well-documented reliability and validity.

Sleep-Related Cognitions.-We used the Dysfunctional Beliefs and Attitudes about Sleep Scale (Carney et al., 2010; Morin, Vallieres, \& Ivers, 2007) to measure maladaptive beliefs about sleep. The dysfunctional beliefs and attitudes about sleep scale is reliable and valid, (Morin et al., 2007) and had an alpha coefficient of 0.89 in this study. A higher score reflects higher dysfunctional beliefs and attitudes about sleep.

The Sleep Disturbance Questionnaire was used to evaluate beliefs about the sources of insomnia (Espie, Inglis, Harvey, \& Tessier, 2000). It includes four factors (restlessness/ agitation, mental over-activity, consequences of insomnia, and lack of sleep readiness). It is reliable and valid (Espie et al., 2000; S. Smith \& Trinder, 2001; Violani, Devoto, Lucidi, Lombardo, \& Russo, 2004) and had a Cronbach's alpha $=0.87$ in the current study.

\section{Statistical analyses}

Actigraph data were downloaded into Actiware v. 5 software (Respironics Minimitter, Inc.). We computed nocturnal sleep duration, efficiency, and sleep latency with standard methods (Redeker et al., 2015). Descriptive statistics were computed for these variables summarized. We had available actigraph data for 49 participants. 
Data were committed to a database. We used logical data checks to identify outliers and data errors after importing the data into SAS and computed descriptive statistics for all variables (frequency distributions of categorical variables and normal distributions for continuous variables). Scatter plots of the daytime-nighttime ratios of the biomarkers were explored to capture outliers, and three extremely large outliers were treated as missing values. Due to skewness, log-transformed biomarker measures were used in the analysis.

We compared demographic and clinical characteristics between the two intervention groups with Wilcoxon, chi-square, and Cochran-Armitage trend tests. We described the biomarkers and the daytime-nighttime ratios at pre- and post-intervention in the CBT-I and attention control conditions with median values ( $1^{\text {st }}$ and $3^{\text {rd }}$ quartiles) that are less influenced by extreme outliers that are commonly observed in biomarkers.

We calculated the means and coefficients of variation of the actigraph-recorded sleep parameters over 3 nights. We calculated partial correlations of the biomarkers with the sleep characteristics and daytime symptoms at pre-intervention after controlling for age.

For the substantive partial correlations (i.e., $|r| \searrow 0.25$ ) at pre-intervention, we confirmed the cross-sectional associations using the general linear mixed model (GLMM), incorporating repeated data collected at pre- and post-intervention with greater powers. The GLMMs for the biomarkers were also controlled for age and outliers were removed from the analysis.

To examine the relationships between the biomarkers and sleep, symptoms, and sleep-related cognitions, we calculated partial correlations between the changed biomarkers and the outcome measures (i.e., $\Delta=$ Value at post-intervention - Value at pre-intervention) after controlling for age. To prevent inflation of type I error, we confirmed the statistical significances of the correlations with the false discovery rate (FDR) of less than 0.05 , which accounts for simultaneous tests with 13 variables with each biomarker ratio (Glickman, Rao, \& Schultz, 2014).

\section{RESULTS}

We previously reported the recruitment process and Consort diagram (Redeker et al., 2015). The sample included 51 participants [CBT-I group $(\mathrm{n}=30)$; attention control group $(\mathrm{n}=$ 21)]. The clinical and demographic characteristics of the sample are presented in table 1. The CBT-I group had higher levels of comorbidity and more diabetes, but fewer participants had pacemakers $(\mathrm{p}<.05)$ than the control group. Five participants in the CBT-I group and 2 in the control group were using positive airway pressure for sleep disordered breathing (Redeker et al., 2015). There were no other statistically significant differences between groups on clinical or demographic characteristics.

Lower median day/night ratios of norepinephrine and epinephrine were observed in the CBT-I compared to the attention control pre-intervention and their medians were consistent at post-intervention (See table 2). However, a higher median day/night ratio of melatonin was observed in the CBT-I group at both pre- and post-intervention. There was no apparent effect of CBT-I on the biomarkers. 
Partial correlations of the log-transformed ratios of the day/night biomarkers with sleep characteristics, daytime symptoms, and physical function at baseline are presented in Table 3. We controlled for age because it was associated with the day/night ratios of the biomarkers $(r=0.12$ to $r=.37)$, with the highest associations between age and the baseline cortisol $(r=0.27, p=.0641)$ and baseline and post-intervention melatonin ratios $(r=0.35, p$ $=.0179)$ and $(\mathrm{r}=0.37, \mathrm{p}=.0161)$, respectively. In the general linear mixed model with repeated data, a higher day/night ratio of cortisol was associated with better sleep quality ( $\mathrm{p}$ $=.0445)$ and less anxiety $(\mathrm{p}=.0182)$, fatigue $(\mathrm{p}=.0194)$, depression $(\mathrm{p}=.0093)$, and negative sleep-related cognitions measured by the sleep disturbance questionnaire $(\mathrm{p}=$. 0023), but not with insomnia, pain, or excessive daytime sleepiness, after controlling for age. The repeated general linear mixed model confirmed the association between the day/night ratio of epinephrine and fatigue $(\mathrm{p}=.0307)$, but did not confirm the association with depression $(\mathrm{p}=.1090)$.

There was a trend suggesting a negative association between the day/night epinephrine ratio and lack of sleep readiness $(r=-0.31, p=.0558)$. The day/night cortisol ratio was negatively associated with mental overactivity $(r=-0.33, p=.0042)$ and the perceived consequences of insomnia $(\mathrm{r}=-0.40, \mathrm{p}=.0130)$, while the melatonin ratio was negatively associated with mental overactivity $(\mathrm{r}=-0.28, \mathrm{p}=.0042)$.

We found substantive partial correlations betweeen the means and coefficients of variation of the actigraph-measured sleep characteristics and epinephrine, cortisol, and melatonin sulfate at pre-intervention, after controlling for age (Table 4). Greater daytime urinary free epinephrine was associated with longer sleep duration $(\mathrm{p}=.0030)$ and sleep efficiency $(\mathrm{p}=$. 0173), while sleep efficiency was associated with the day/night ratio of epinephrine. Daily variability in the coefficients of variation in sleep latency and duration were associated with a greater day/night ratio of melatonin $(\mathrm{p}=.0137)$ and a lower day/night ratio of cortisol (. 0117), respectively.

Changes in the norepinephrine and cortisol ratios had substantive correlations with daytime symptoms and sleep characteristics (Table 5). The partial correlations consistently show that the increased day/night ratio of norepinephrine is associated with improvement in daytime symptoms (e.g. pain, sleepiness, depression) and physical function after controlling for age, although all associations were not statistically significant. The increased ratio of day/night cortisol is associated with improved daytime symptoms (ie, fatigue and depression) and physical function.

The change in the cortisol ratio had substantive correlations with improvement in sleep quality, sleep duration, and sleep-related cognitions. Based on the false discovery rate (FDR), only the correlations between the changed ratio of cortisol and fatigue (FDR $=$. 0024), depression (FDR $=.0220)$, and sleep duration (FDR $=.0220)$ were statistically significant. The ratio of day/night epinephrine was inversely associated with restlessness/ agitation, and mental overactivity, while increases in the ratio of day/night cortisol level were associated with decreases in restlessness/agitation, mental overactivity, and the consequences of insomnia. 


\section{DISCUSSION}

To our knowledge, this is the first study to explore the effects of CBT-I on day and night biomarkers of the hypothalamic pituitary axis and autonomic function among patients with $\mathrm{HF}$, a group that is vulnerable to the negative effects of alterations in these biological pathways. There were no detectable direct effects of CBT-I on these biomarkers. However, the substantive cross-sectional correlations between sleep disturbance, objective sleep characteristics, and biomarkers, especially cortisol, suggest that these symptoms are associated with biological stress. Moderate to large and statistically significant associations between changes in urinary cortisol ratios and sleep disturbance and fatigue suggest that alterations in the HPA Axis might contribute to improved symptoms. However, the extent to which these changes are secondary to improvements in sleep characteristics cannot be determined from these data.

Although insomnia severity was not associated with the biomarkers in this study, the negative association between the sleep disturbance questionnaire total score and subscales and the epinephrine and cortisol ratios at baseline, as well as the association between improvements in cognitions and the cortisol and norepinephrine levels, support the close connection between sleep-related cognitions and the hypothalamic pituitary axis and autonomic function. Taken together with our previously reported findings of statistically significant effects of CBT-I on insomnia, sleep disturbance and fatigue and dysfunctional beliefs and cognitions about sleep (Redeker et al., 2015; Redeker et al., 2017), these findings suggest that CBT-I may contribute to both biological and cognitive effects on hyperarousal, a phenomenon associated with sympathetic activation among HF patients. Given the deleterious consequences of nocturnal sympathetic activation in HF patients, the potential beneficial effects of CBT-I on psychophysiologic arousal among patients with HF warrant further examination in a larger study.

The ratio of day/night melatonin was negatively associated with day to day variability in sleep latency and with mental overactivity. However, given that normal melatonin levels are higher at night than during the day, these findings were in the opposite direction to what might be expected and may reflect excessive exposure to light at night that would decrease nocturnal melatonin levels, decreased time in bed associated with the sleep restriction component of CBT-I and/or the confounding effects of beta blocker medications commonly taken by HF patients that may decrease melatonin levels (Fares, 2011). Although we screened participants for use of exogenous melatonin, it is possible that unreported use of this supplement over the course of the study may have influenced the findings. These findings merit further exploration in a future larger study.

Notably, there was little difference between the day and night melatonin sulfate levels in the CBT-I group. These findings may suggest that in addition to insomnia, people with HF have circadian dysfunction and may benefit from circadian interventions, such as timed bright light therapy or exogenous supplementation of melatonin, in addition to CBT-I.

Researchers and clinicians are just beginning to understand the importance of melatonin to patients with HF. While the effects of endogenous melatonin on biological rhythms are well- 
documented, and studies in other populations documented associations between melatonin rhythm and fatigue (Kwon et al., 2015; Melamud, Golan, Luboshitzky, Lavi, \& Miller, 2012), melatonin also has anti-oxidant (Reiter et al., 2016), anti-inflammatory (DominguezRodriguez, Abreu-Gonzalez, Sanchez-Sanchez, Kaski, \& Reiter, 2010), and cardioprotective properties, including reductions in excessive heart rate through inhibitory effects on the sympathetic nervous system (Simko, Baka, Paulis, \& Reiter, 2016). Day and night levels of melatonin were higher among patients with New York Heart Association Class II than Class II HF and inversely related to brain natriuretic peptide (BNP) levels (Dzida et al., 2013). Lower melatonin levels were also associated with reduced cardiac remodeling and survival in rats (Simko et al., 2014), and diurnal melatonin levels were significantly lower among HF patients who did not respond with reversed left ventricular remodelling to cardiac resynchronization therapy (Dominguez-Rodriguez, Abreu-Gonzalez, Piccolo, Galasso, \& Reiter, 2016). Research is needed to further explore circadian function and potential interventions to improve the melatonin profiles in people with HF and to determine if positive changes in melatonin profiles also result in improved HF outcomes.

Limitations. The parent study was designed to evaluate the feasibility, acceptability, and effect size of CBT-I on insomnia and daytime symptoms in preparation for a larger fully powered clinical trial, and the biomarker assessment was exploratory in nature. Thus, the study was not powered to determine statistically significant effects of treament on the biomarkers.

To enable comparison of day and night levels, we obtained urinary measures of the biomarkers during the night and over the afternoon from 1-5 PM, but we did not obtain serial measures that would enable calculation of the cortisol awakening response or dim light melatonin onset that would have allowed a more granular interpretation of the results. Given that cortisol is generally highest upon awakening, afternoon measurement may have resulted in under-estimation of daytime levels. Although we screened patients for use of cortisone containing medications and exogenous melatonin intake, HF patients in this study had high degrees of comorbidity and many prescribed medications (e.g., beta blockers and other adrenergic drugs) that may have influenced the the biomarkers.

Research on sleep and its effects among people with HF is challenging due to the comorbid influences on sleep and sleep disorders and the high degree of sleep fragmentation associated with sleep disordered breathing and other issues (Redeker, Muench, et al., 2010; Turoff et al., 2017). Comorbid sleep disorders, as well as HF itself may influence sleep continuity and the biomarkers measured in the current study. Although in our previous work in a group of stable HF patients (Redeker, Jeon, et al., 2010; Redeker, Muench, et al., 2010), sleep disordered breathing did not explain insomnia symptoms, self-reported sleep quality, or daytime symptoms, we addressed the potential influences of these primary sleep disorders on the study variables by including patients with no more than mild sleep disordered breathing or those who were adherent to positive airway pressure treatment. We did not include patients with known restless legs syndrome, or narcolepsy.

We did not distinguish between patients with HF with preserved vs. reduced ejection fraction because there was no difference in insomnia based on these HF characteristics in 
our previous work (Redeker, Jeon, et al., 2010) and do not have data available on ejection fraction for this study. Yet, it is possible that differences in HF or comorbidity between CBT-I and attention-control groups or unmeasured differences in HF contributed to our findings.. Other HF and cardiovascular characteristics (e.g., dysrhythmias) should be considered in more detail in future studies. For example, recent evidence suggest that atrial fibrillation contributes to insomnia (Chokesuwattanaskul et al., 2018; Christensen et al., 2018; Han et al., 2017; Lee et al., 2017).

The attention control group included standard patient education for HF with the assumption that the HF information would already be familiar to these patients who received care in evidence-based HF disease management programs and was designed to control for therapist time and attention. It did not include active behavioral change strategies, in comparison to the CBT-I group. It is possible, but we think unlikely, that the HF education influenced HF self-management behaviora and influenced the biomarkers.

Taken together, the findings of this study suggest potentially important relationships between sleep disturbance, sleep characteristics, sleep-related symptoms and cognitions and biomarkers important to HF disease progression, especially cortisol. Future, more fully powered studies are needed to evaluate the extent to which CBT-I or pharmacologic treatment of insomnia leads to improvement in pathological pathways shared by insomnia and HF and potential improvement in HF-related morbidity and mortality.

\section{References}

Ahmad T, Fiuzat M, Felker GM, \& O’Connor C (2012). Novel biomarkers in chronic heart failure. Nature Reviews Cardiology, 9(6), 347-359. doi: 10.1038/nrcardio.2012.37 [PubMed: 22450126]

Anderson GM, Durkin TA, Morton JB, \& Cohen DJ (1988). Liquid chromatographic determination of urinary catecholamines after one-step alumina extraction. Journal of Chromatography, 424(2), 373377. [PubMed: 3372630]

Backhaus J, Junghanns K, \& Hohagen F (2004). Sleep disturbances are correlated with decreased morning awakening salivary cortisol. Psychoneuroendocrinology, 29(9), 1184-1191. doi: 10.1016/ j.psyneuen.2004.01.010 [PubMed: 15219642]

Basta M, Chrousos GP, Vela-Bueno A, \& Vgontzas AN (2007). Chronic Insomnia and Stress System. Sleep Medicine Clinics, 2(2), 279-291. doi: 10.1016/j.jsmc.2007.04.002 [PubMed: 18071579]

Bastien CH, Vallieres A, \& Morin CM (2001). Validation of the Insomnia Severity Index as an outcome measure for insomnia research. Sleep Medicine, 2(4), 297-307. [PubMed: 11438246]

Belza BL (1990). Multidimensional Assessment of Fatigue (MAF) Scale Users Guide (pp. 1-3). Seattle: University of Washington.

Benjamin EJ, Blaha MJ, Chiuve SE, Cushman M, Das SR, Deo R, ... Stroke Statistics S (2017). Heart Disease and Stroke Statistics-2017 Update: A Report From the American Heart Association. Circulation, 135(10), e146-e603. doi: 10.1161/CIR.0000000000000485 [PubMed: 28122885]

Benson K, Friedman L, Noda A, Wicks D, Wakabayashi E, \& Yesavage J (2004). The measurement of sleep by actigraphy: direct comparison of 2 commercially available actigraphs in a nonclinical population. Sleep, 27(5), 986-989. [PubMed: 15453559]

Blais FC, Gendron L, Mimeault V, \& Morin CM (1997). [Evaluation of insomnia: validity of 3 questionnaires]. Encephale, 23(6), 447-453. [PubMed: 9488928]

Blood MR, Sack RL, Percy DC, \& Pen J (1997). A comparison of sleep detection by wrist actigraph, behavioral response, and polysomnography. Sleep, 20, 388-395. [PubMed: 9302721] 
Buysse DJ, Reynolds CF 3rd, Monk TH, Berman SR, \& Kupfer DJ (1989). The Pittsburgh Sleep Quality Index: a new instrument for psychiatric practice and research. Psychiatry Research, 28(2), 193-213. [PubMed: 2748771]

Buysse DJ, Reynolds CF, Monk TH, Hoch CC, Yeager AL, \& Kupfer DJ (1991). Quantification of subjective sleep quality in healthy elderly men and women using the Pittsburgh Sleep Quality Index (PSQI). Sleep, 14, 331-338. [PubMed: 1947597]

Carney CE, Edinger JD, Morin CM, Manber R, Rybarczyk B, Stepanski EJ, ... Lack L (2010). Examining maladaptive beliefs about sleep across insomnia patient groups. Journal of Psychosomatic Resesarch, 68(1), 57-65. doi: 10.1016/j.jpsychores.2009.08.007

Carroll JE, Seeman TE, Olmstead R, Melendez G, Sadakane R, Bootzin R, ... Irwin MR (2015). Improved sleep quality in older adults with insomnia reduces biomarkers of disease risk: Pilot results from a randomized controlled comparative efficacy trial. Psychoneuroendocrinology, 55, 184-192. doi: 10.1016/j.psyneuen.2015.02.010 [PubMed: 25770704]

Charlson ME, Pompei P, Ales KL, \& MacKenzie CR (1987). A new method of classifying prognostic comorbidity in longitudinal studies: development and validation. J Chronic Disease, 40(5), 373383. [PubMed: 3558716]

Chokesuwattanaskul R, Thongprayoon C, Sharma K, Congrete S, Tanawuttiwat T, \& Cheungpasitporn $\mathrm{W}$ (2018). Associations of sleep quality with incident atrial fibrillation: a meta-analysis. Internal Medicine Journal, 48(8), 964-972. doi: 10.1111/imj.13764 [PubMed: 29460411]

Christensen MA, Dixit S, Dewland TA, Whitman IR, Nah G, Vittinghoff E, ... Marcus GM (2018). Sleep characteristics that predict atrial fibrillation. Heart Rhythm. doi: 10.1016/j.hrthm. 2018.05.008

Devins G, \& Orme C (1985). Center for Epidemiologic Studies Depression Scale In Keyser D \& Sweetland R (Eds.), Test Critiques (pp. 144-160). Kansas City, MO: Test Corporation of American, a susidiary of Westport Publishers, Inc.

Dominguez-Rodriguez A, Abreu-Gonzalez P, Piccolo R, Galasso G, \& Reiter RJ (2016). Melatonin is associated with reverse remodeling after cardiac resynchronization therapy in patients with heart failure and ventricular dyssynchrony. International Journal of Cardiology, 221, 359-363. doi: 10.1016/j.ijcard.2016.07.056 [PubMed: 27404706]

Dominguez-Rodriguez A, Abreu-Gonzalez P, Sanchez-Sanchez JJ, Kaski JC, \& Reiter RJ (2010). Melatonin and circadian biology in human cardiovascular disease. Journal of Pineal Research, 49(1), 14-22. doi: 10.1111/j.1600-079X.2010.00773.x [PubMed: 20536686]

Dzida G, Prystupa A, Lachowska-Kotowska P, Kadas T, Kamienski P, Kimak E, ... Kicinski P (2013). Alteration in diurnal and nocturnal melatonin serum level in patients with chronic heart failure. Annals of Agricultural \& Environmental Medicine, 20(4), 745-748. [PubMed: 24364447]

Edinger JD (2004). Classifying insomnia in a clinically useful way. Journal of Clinical Psychiatry, 65 Suppl 8, 36-43.

Edinger JD, Wohlgemuth WK, Radtke RA, Coffman CJ, \& Carney CE (2007). Dose-response effects of cognitive-behavioral insomnia therapy: a randomized clinical trial. Sleep, 30(2), 203-212. [PubMed: 17326546]

Espie CA, Inglis SJ, Harvey L, \& Tessier S (2000). Insomniacs' attributions. psychometric properties of the Dysfunctional Beliefs and Attitudes about Sleep Scale and the Sleep Disturbance Questionnaire. Journal of Psychosomatic Research, 48(2), 141-148. [PubMed: 10719130]

Fares A (2011). Night-time exogenous melatonin administration may be a beneficial treatment for sleeping disorders in beta blocker patients. Journal of Cardiovascular Disease Research, 2(3), 153155. doi: 10.4103/0975-3583.85261 [PubMed: 22022142]

Gallucci M, Flores-Obando R, Mazzuco S, Ongaro F, Di Giorgi E, Boldrini P, ... Taioli E (2014). Melatonin and the Charlson Comorbidity Index (CCI). International Journal of Biological Markers, 29(3), e253-260. doi: 10.5301/jbm.5000077 [PubMed: 24557791]

Gau FY, Chen XP, Wu HY, Lin ML, \& Chao YF (2011). Sleep-related predictors of quality of life in the elderly versus younger heart failure patients: a questionnaire survey. International Journal of Nursing Studies, 48(4), 419-428. doi: 10.1016/j.ijnurstu.2010.07.011 [PubMed: 20696428] 
Han X, Yang Y, Chen Y, Gao L, Yin X, Li H, ... Xia Y (2017). Association between insomnia and atrial fibrillation in a Chinese population: A cross-sectional study. Clinical Cardiology, 40(9), 765769. doi: 10.1002/clc.22731 [PubMed: 28561943]

Irwin MR, Olmstead R, Breen EC, Witarama T, Carrillo C, Sadeghi N, ... Cole S (2015). Cognitive behavioral therapy and tai chi reverse cellular and genomic markers of inflammation in late-life insomnia: a randomized controlled trial. Biological Psychiatry, 78(10), 721-729. doi: 10.1016/ j.biopsych.2015.01.010 [PubMed: 25748580]

Irwin MR, Olmstead R, Carrillo C, Sadeghi N, Breen EC, Witarama T, ... Nicassio P (2014). Cognitive behavioral therapy vs. Tai Chi for late life insomnia and inflammatory risk: a randomized controlled comparative efficacy trial. Sleep, 37(9), 1543-1552. doi: 10.5665/sleep. 4008 [PubMed: 25142571]

Jeon S, \& Redeker NS (2016). Sleep Disturbance, Daytime Symptoms, and Functional Performance in Patients With Stable Heart Failure: A Mediation Analysis. Nursing Research, 65(4), 259-267. doi: 10.1097/NNR.0000000000000169 [PubMed: 27362512]

Johns MW (1991). A new method for measuring daytime sleepiness: the Epworth sleepiness scale. Sleep, 14(6), 540-545. [PubMed: 1798888]

Kanno Y, Yoshihisa A, Watanabe S, Takiguchi M, Yokokawa T, Sato A, ... Takeishi Y(2016). Prognostic Significance of Insomnia in Heart Failure. Circulation Journal, 80(7), 1571-1577. doi: 10.1253/circj.CJ-16-0205 [PubMed: 27194467]

Kushida CA, Chang A, Gadkary C, Guilleminault C, Carrillo O, \& Dement WC (2001). Comparison of actigraphic, polysomnographic, and subjective assessment of sleep parameters in sleepdisordered patients. Sleep Medicine, 2(5), 389-396. [PubMed: 14592388]

Kwon KJ, Lee EJ, Kim MK, Jeon SJ, Choi YY, Shin CY, \& Han SH (2015). The potential role of melatonin on sleep deprivation-induced cognitive impairments: implication of FMRP on cognitive function. Neuroscience, 301, 403-414. doi: 10.1016/j.neuroscience.2015.05.079 [PubMed: 26047724]

Lack LC, Gradisar M, Van Someren EJ, Wright HR, \& Lushington K (2008). The relationship between insomnia and body temperatures. Sleep Medicine Reviews, 12(4), 307-317. doi: 10.1016/j.smrv. 2008.02.003 [PubMed: 18603220]

Laugsand LE, Strand LB, Platou C, Vatten LJ, \& Janszky I (2014). Insomnia and the risk of incident heart failure: a population study. European Heart Journal, 35(21), 1382-1393. doi: 10.1093/ eurheartj/eht019 [PubMed: 23462728]

Lee HH, Chen YC, Chen JJ, Lo SH, Guo YL, \& Hu HY (2017). Insomnia and the Risk of Atrial Fibrillation: A Population-Based Cohort Study. Acta Cardiologica Sinica, 33(2), 165-172. [PubMed: 28344420]

Lichstein KL, Stone KC, Donaldson J, Nau SD, Soeffing JP, Murray D, ... Aguillard RN (2006). Actigraphy validation with insomnia. Sleep, 29(2), 232-239. [PubMed: 16494091]

Littner M, Kushida CA, Anderson WM, Bailey D, Berry RB, Davila DG, ... Standards of Practice Committee of the American Academy of Sleep, M. (2003). Practice parameters for the role of actigraphy in the study of sleep and circadian rhythms: an update for 2002. Sleep, 26(3), 337-341. [PubMed: 12749556]

Meerlo P, Sgoifo A, \& Suchecki D (2008). Restricted and disrupted sleep: effects on autonomic function, neuroendocrine stress systems and stress responsivity. Sleep Medicine Reviews, 12(3), 197-210. doi: 10.1016/j.smrv.2007.07.007 [PubMed: 18222099]

Melamud L, Golan D, Luboshitzky R, Lavi I, \& Miller A (2012). Melatonin dysregulation, sleep disturbances and fatigue in multiple sclerosis. Journal of Neurological Science, 314(1-2), 37-40. doi: 10.1016/j.jns.2011.11.003

Miller CB, Kyle SD, Gordon CJ, Espie CA, Grunstein RR, Mullins AE, ... Bartlett DJ (2015). Physiological Markers of Arousal Change with Psychological Treatment for Insomnia: A Preliminary Investigation. PLoS One, 10(12), e0145317. doi: 10.1371/journal.pone.0145317 [PubMed: 26683607]

Morin CM, Belleville G, Belanger L, \& Ivers H (2011). The Insomnia Severity Index: psychometric indicators to detect insomnia cases and evaluate treatment response. Sleep, 34(5), 601-608. [PubMed: 21532953] 
Morin CM, Vallieres A, \& Ivers H (2007). Dysfunctional beliefs and attitudes about sleep (DBAS): validation of a brief version (DBAS-16). Sleep, 30(11), 1547-1554. [PubMed: 18041487]

Pereg D, Chan J, Russell E, Berlin T, Mosseri M, Seabrook JA, ... Van Uum S (2013). Cortisol and testosterone in hair as biological markers of systolic heart failure. Psychoneuroendocrinology, 38(12), 2875-2882. doi: 10.1016/j.psyneuen.2013.07.015 [PubMed: 23972942]

Radloff LS, \& Teri L (1986). Use of the Center for Epidemiological Studies-Depression Scale with older adults. Clinical Gerontology, 5(1/2), 119-136.

Redeker NS, \& Hilkert R (2005). Sleep and quality of life in stable heart failure. Journal of Cardiac Failure, 11(9), 700-704. doi: 10.1016/j.cardfail.2005.07.003 [PubMed: 16360966]

Redeker NS, Jeon S, Andrews L, Cline J, Jacoby D, \& Mohsenin V (2015). Feasibility and Efficacy of a Self-Management Intervention for Insomnia in Stable Heart Failure. Journal of Clinical Sleep Medicine, 11(10), 1109-1119. doi: 10.5664/jcsm.5082 [PubMed: 25979100]

Redeker NS, Jeon S, Andrews L, Cline J, Mohsenin V, \& Jacoby D (2017). Effects of Cognitive Behavioral Therapy for Insomnia on Sleep-Related Cognitions Among Patients With Stable Heart Failure. Behavioral Sleep Medicine, 1-13. doi: 10.1080/15402002.2017.1357120

Redeker NS, Jeon S, Muench U, Campbell D, Walsleben J, \& Rapoport DM (2010). Insomnia symptoms and daytime function in stable heart failure. Sleep, 33(9), 1210-1216. [PubMed: 20857868]

Redeker NS, Muench U, Zucker MJ, Walsleben J, Gilbert M, Freudenberger R, ... Rapoport DM (2010). Sleep disordered breathing, daytime symptoms, and functional performance in stable heart failure. Sleep, 33(4), 551-560. [PubMed: 20394325]

Redeker NS, \& Stein S (2006). Characteristics of sleep in patients with stable heart failure versus a comparison group. Heart \& Lung, 35(4), 252-261. doi: 10.1016/j.hrtlng.2005.10.007 [PubMed: 16863897]

Reiter RJ, Mayo JC, Tan DX, Sainz RM, Alatorre-Jimenez M, \& Qin L (2016). Melatonin as an antioxidant: under promises but over delivers. Journal of Pineal Research, 61(3), 253-278. doi: 10.1111/jpi.12360 [PubMed: 27500468]

Sadeh A (2011). The role and validity of actigraphy in sleep medicine: an update. Sleep Medicine Reviews, 15(4), 259-267. doi: 10.1016/j.smrv.2010.10.001 [PubMed: 21237680]

Savard J, Simard S, Ivers H, \& Morin CM (2005). Randomized study on the efficacy of cognitivebehavioral therapy for insomnia secondary to breast cancer, part II: Immunologic effects. Journal of Clinical Oncology, 23(25), 6097-6106. doi: 10.1200/JCO.2005.12.513 [PubMed: 16135476]

Simko F, Baka T, Paulis L, \& Reiter RJ (2016). Elevated heart rate and nondipping heart rate as potential targets for melatonin: a review. Journal of Pineal Research, 61(2), 127-137. doi: 10.1111/ jpi.12348 [PubMed: 27264986]

Simko F, Bednarova KR, Krajcirovicova K, Hrenak J, Celec P, Kamodyova N, ... Adamcova M (2014). Melatonin reduces cardiac remodeling and improves survival in rats with isoproterenolinduced heart failure. Journal of Pineal Research, 57(2), 177-184. doi: 10.1111/jpi.12154 [PubMed: 24942291]

Smith MT, Huang MI, \& Manber R (2005). Cognitive behavior therapy for chronic insomnia occurring within the context of medical and psychiatric disorders. Clinical Psychology Reviews, 25(5), 559592. doi: 10.1016/j.cpr.2005.04.004

Smith S, \& Trinder J (2001). Detecting insomnia: comparison of four self-report measures of sleep in a young adult population. Journal of Sleep Research, 10(3), 229-235. [PubMed: 11696076]

Spielberger CD (1983). Manual for the State-Trait Anxiety Inventory STAI-Form Y. Palo Alto, CA: Consulting Psychologists Press.

Tsikas D, Wolf A, \& Frolich JC (2004). Simplified HPLC method for urinary and circulating creatinine. Clinical Chemistry, 50(1), 201-203. doi: 10.1373/clinchem.2003.024141 [PubMed: 14709648]

Turoff A, Thiem U, Fox H, Spiesshofer J, Bitter T, Tamisier R, ... Oldenburg O (2017). Sleep duration and quality in heart failure patients. Sleep \& Breathing. doi: 10.1007/s11325-017-1501-X

Vgontzas AN, \& Chrousos GP (2002). Sleep, the hypothalamic-pituitary-adrenal axis, and cytokines: multiple interactions and disturbances in sleep disorders. Endocrinology \& Metabolism Clinics of North America, 31(1), 15-36. [PubMed: 12055986] 
Vgontzas AN, Zoumakis M, Papanicolaou DA, Bixler EO, Prolo P, Lin HM, ... Chrousos GP (2002). Chronic insomnia is associated with a shift of interleukin- 6 and tumor necrosis factor secretion from nighttime to daytime. Metabolism, 51(7), 887-892. doi: S0026049502000148 [pii] [PubMed: 12077736]

Violani C, Devoto A, Lucidi F, Lombardo C, \& Russo PM (2004). Validity of a short insomnia questionnaire: the SDQ. Brain Research Bulletin, 63(5), 415-421. doi: 10.1016/j.brainresbull. 2003.06.002 [PubMed: 15245769]

Westgard JO, Barry PL, Hunt MR, \& Groth T (1981). A multi-rule Shewhart chart for quality control in clinical chemistry. Clinical Chemistry, 27(3), 493-501. [PubMed: 7471403]

Wong ML, Lau KNT, Espie CA, Luik AI, Kyle SD, \& Lau EYY (2017). Psychometric properties of the Sleep Condition Indicator and Insomnia Severity Index in the evaluation of insomnia disorder. Sleep Medicine, 33, 76-81. doi: 10.1016/j.sleep.2016.05.019 [PubMed: 28449911] 
Table 1.

Demographic and Clinical Characteristics of the Sample

\begin{tabular}{|c|c|c|c|c|}
\hline Variables & $\begin{array}{c}\text { Overall } \\
\mathrm{N}=\mathbf{5 1}\end{array}$ & $\begin{array}{r}\text { CBT-I } \\
\mathbf{N}=30\end{array}$ & $\begin{array}{r}\text { Attention Control } \\
\mathbf{N}=21\end{array}$ & P-value \\
\hline Age & $59.1(15.1)$ & $62.0(13.1)$ & $55.0(17.1)$ & (1) .1800 \\
\hline Male & $25(49.0 \%)$ & $16(53.3 \%)$ & $10(47.6 \%)$ & ${ }^{(2)} .6879$ \\
\hline White & $34(66.7 \%)$ & $19(63.3 \%)$ & $15(71.4 \%)$ & (2). .5461 \\
\hline \multicolumn{5}{|l|}{ NYHA } \\
\hline I & $2(4.1 \%)$ & $2(6.7 \%)$ & $0(0 \%)$ & (3) 6709 \\
\hline II & $29(59.2 \%)$ & $17(56.7 \%)$ & $12(63.2 \%)$ & \\
\hline III & $18(36.7 \%)$ & $11(36.7 \%)$ & $7(36.8 \%)$ & \\
\hline Charlson Comorbidity Index & $2.2(1.5)$ & $2.5(1.5)$ & $1.7(1.3)$ & (1).0213 \\
\hline Diabetes & $16(31.4 \%)$ & $13(43.3 \%)$ & $3(14.3 \%)$ & (4). 0352 \\
\hline Angina & $19(37.2 \%)$ & $8(26.7 \%)$ & $11(52.4 \%)$ & (4).0815 \\
\hline Hypertension & $39(78.0 \%$ & $25(83.3 \%)$ & $14(70 \%)$ & (4) 3109 \\
\hline Myocardial Infarction & $14(28.6 \%)$ & $9(32.1 \%)$ & $5(23.8 \%)$ & (4).7502 \\
\hline Pacemaker & $18(35.3 \%)$ & $6(20 \%)$ & $12(57.1 \%)$ & (4).0086 \\
\hline Internal Cardiac Defibrillator & $18(35.3 \%)$ & $8(26.7 \%)$ & $10(47.6 \%)$ & (4) 1469 \\
\hline Peripheral Edema & $23(46 \%)$ & $14(48.3 \%)$ & $9(42.9 \%)$ & (4).7785 \\
\hline "irregular heart beat" & $39(78 \%)$ & $21(72.4 \%)$ & $18(85.7 \%)$ & (4) .3186 \\
\hline Chronic Obstructive Pulmonary Disease & $8(15.7 \%)$ & $7(23.3 \%)$ & $1(4.8 \%)$ & (4) 1192 \\
\hline Depression & $12(23.5 \%)$ & $7(23.3 \%)$ & $5(23.8 \%)$ & (4) 1.000 \\
\hline
\end{tabular}

In group comparison tests, p-values are obtained from Wilcoxon rank test (1), chi-square test (2), Cochran-Armitage Trend test (3), and Fisher's Exact test (4) 


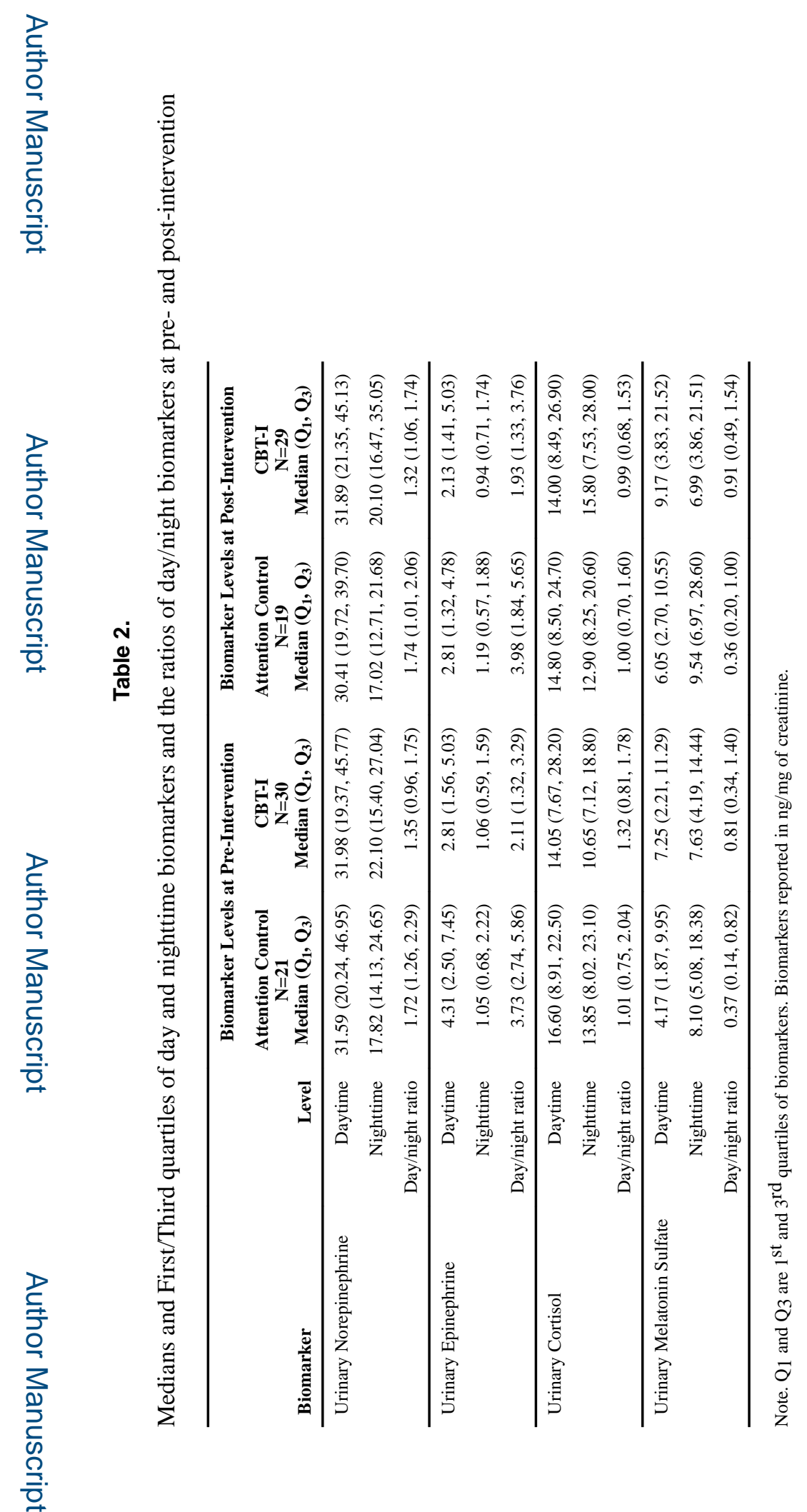


Redeker et al.

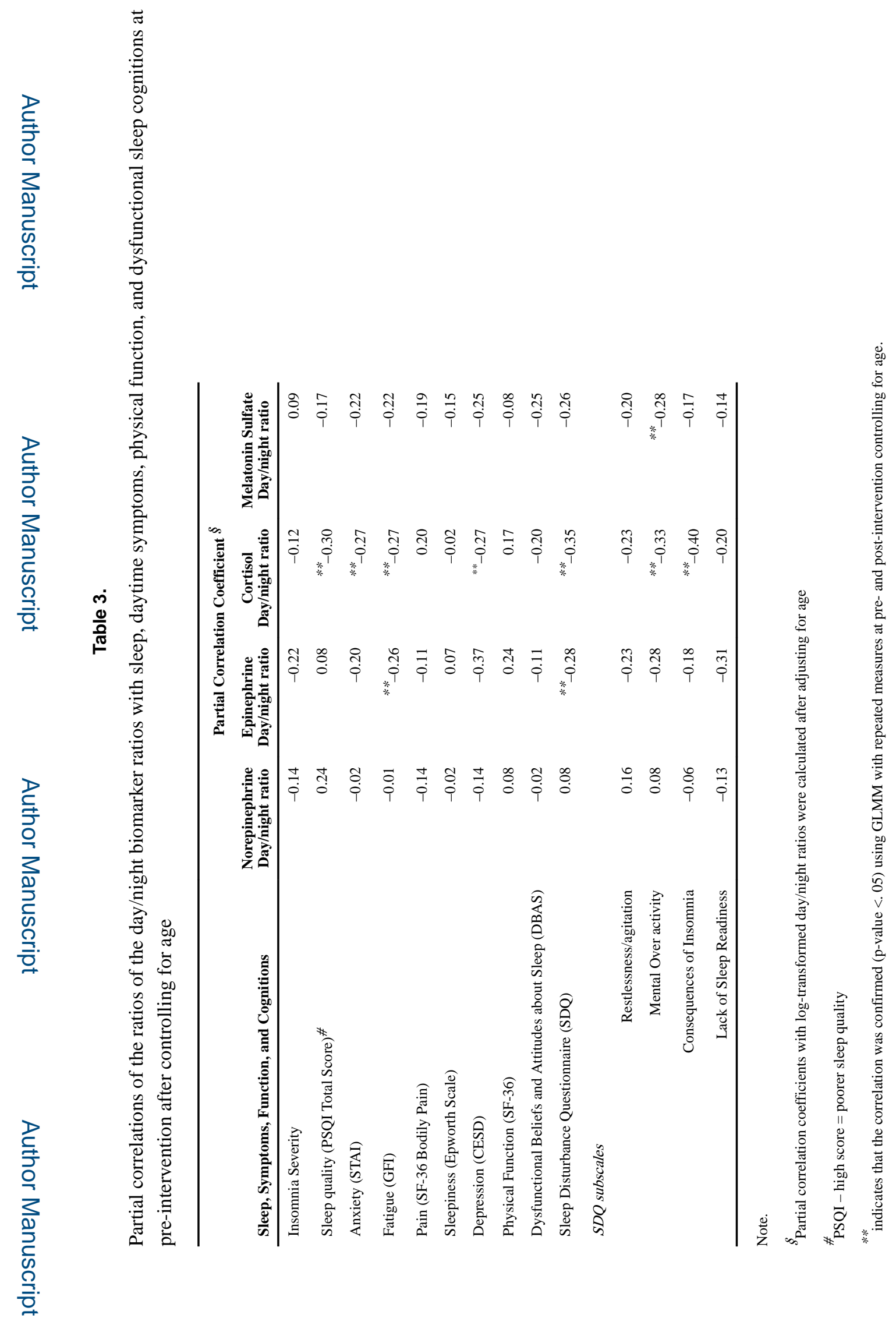

Behav Sleep Med. Author manuscript; available in PMC 2020 May 21. 


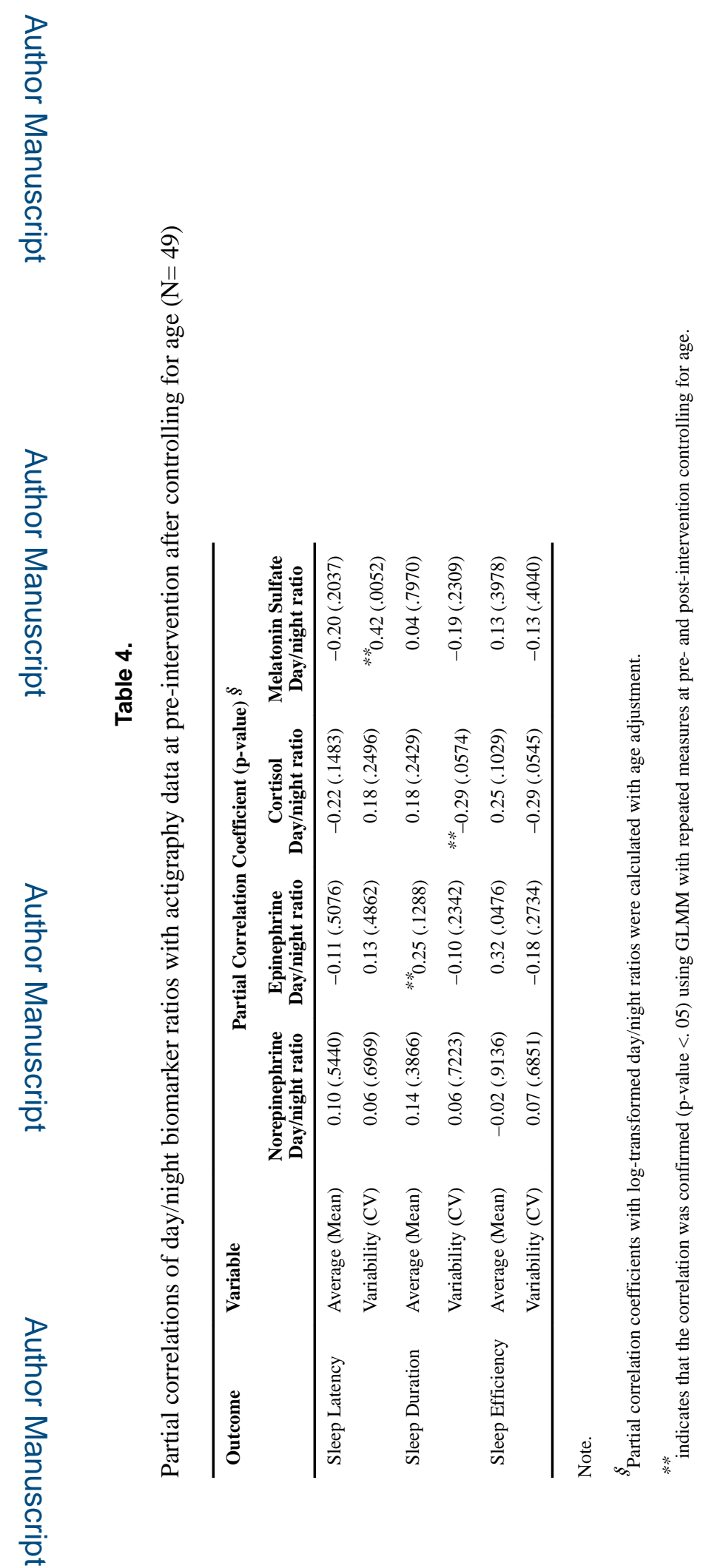


Redeker et al.

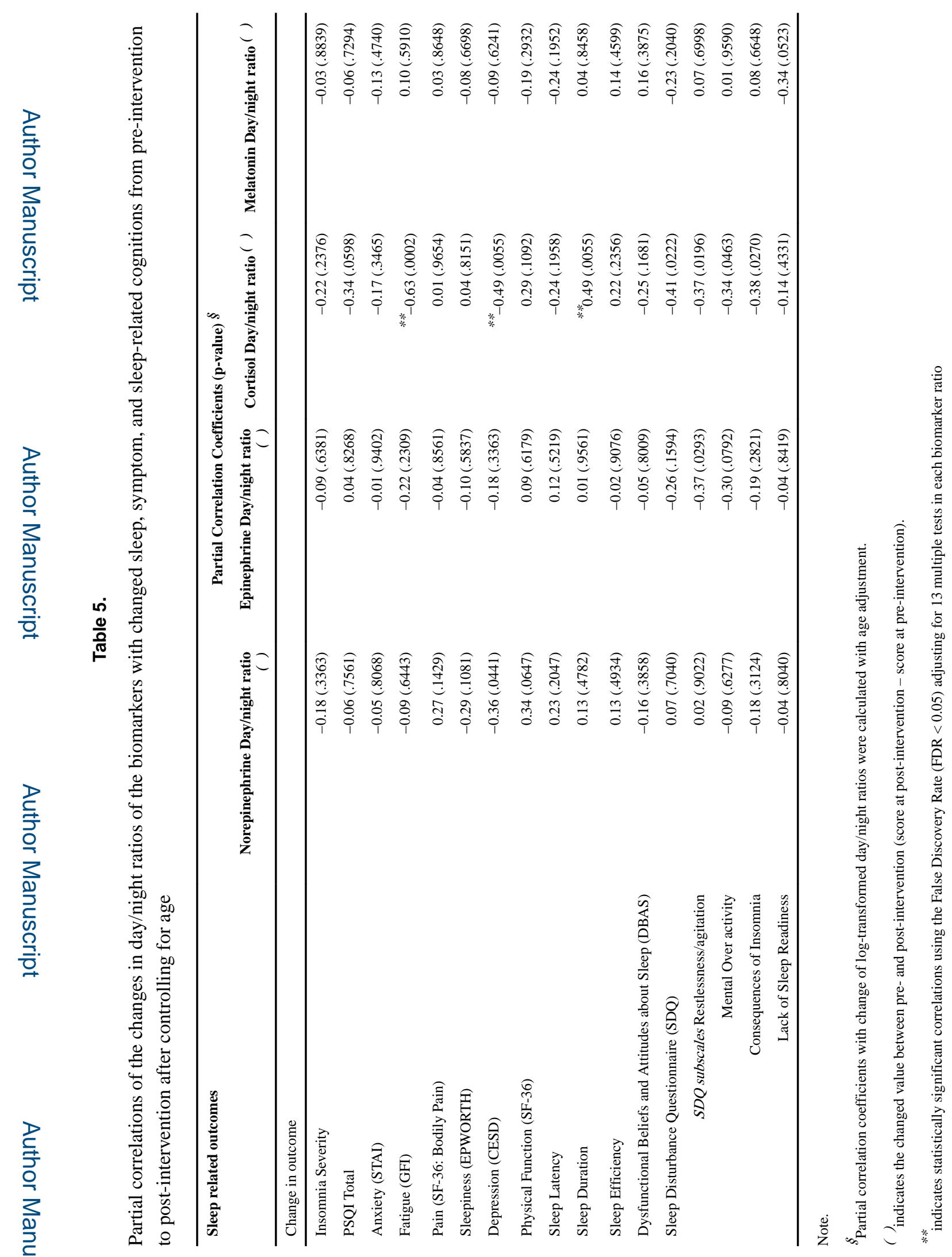

Behav Sleep Med. Author manuscript; available in PMC 2020 May 21. 